\title{
Safety assessment of spine MRI in deep brain stimulation patients
}

\author{
Alexandre Boutet, MD, MSc, ${ }^{1,2}$ Gavin J. B. Elias, BA, ${ }^{2}$ Robert Gramer, BSc, ${ }^{2}$ \\ Clemens Neudorfer, MD, ${ }^{2}$ Jürgen Germann, MSc, ${ }^{2}$ Asma Naheed, MRT, ${ }^{1}$ Nicole Bennett, MRT, \\ Bryan Li, MRT, ${ }^{2}$ Dave Gwun, ${ }^{2}$ Clement T. Chow, BKin, ${ }^{2}$ Ricardo Maciel, MD, MSc, ${ }^{2,3}$ \\ Alejandro Valencia, NP, ${ }^{2}$ Alfonso Fasano, MD, PhD, ${ }^{3,4}$ Renato P. Munhoz, MD, PhD, ${ }^{3,4}$ \\ Warren Foltz, PhD, ${ }^{5}$ David Mikulis, MD, ${ }^{1,2,4}$ Ileana Hancu, PhD, ${ }^{6}$ Suneil K. Kalia, MD, PhD, ${ }^{2}$ \\ Mojgan Hodaie, MD, MSc, ${ }^{2}$ Walter Kucharczyk, MD, ${ }^{1,2}$ and Andres M. Lozano, MD, PhD²
}

${ }^{1}$ Joint Department of Medical Imaging, University of Toronto; ${ }^{2}$ University Health Network, Toronto; ${ }^{3}$ Edmond J. Safra Program in Parkinson's Disease, Morton and Gloria Shulman Movement Disorders Clinic, Toronto Western Hospital, University Health Network, Division of Neurology, University of Toronto; ${ }^{4}$ Krembil Brain Institute, Toronto; ${ }^{5}$ Department of Radiation Oncology, STTARR Innovation Centre, University Health Network, Toronto, Ontario, Canada; and ${ }^{6}$ National Institutes of Health, Center for Scientific Review, Bethesda, Maryland

OBJECTIVE Many centers are hesitant to perform clinically indicated MRI in patients who have undergone deep brain stimulation (DBS). Highly restrictive guidelines prohibit the use of most routine clinical MRI protocols in these patients. The authors' goals were to assess the safety of spine MRI in patients with implanted DBS devices, first through phantom model testing and subsequently through validation in a DBS patient cohort.

METHODS A phantom was used to assess DBS device heating during 1.5-T spine MRI. To establish a safe spine protocol, routinely used clinical sequences deemed unsafe (a rise in temperature $>2^{\circ} \mathrm{C}$ ) were modified to decrease the rise in temperature. This safe phantom-based protocol was then used to prospectively run 67 spine MRI sequences in 9 DBS participants requiring clinical imaging. The primary outcome was acute adverse effects; secondary outcomes included long-term adverse clinical effects, acute findings on brain MRI, and device impedance stability.

RESULTS The increases in temperature were highest when scanning the cervical spine and lowest when scanning the lumbar spine. A temperature rise $<2^{\circ} \mathrm{C}$ was achieved when $3 \mathrm{D}$ sequences were modified to $2 \mathrm{D}$ and when the number of slices was decreased by the minimum amount compared to routine spine MRI protocols (but there were still more slices than allowed by vendor guidelines). Following spine MRI, no acute or long-term adverse effects or acute findings on brain MR images were detected. Device impedances remained stable.

CONCLUSIONS Patients with DBS devices may safely undergo spine MRI with a fewer number of slices compared to those used in routine clinical protocols. Safety data acquisition may allow protocols outside vendor guidelines with a maximized number of slices, reducing the need for radiologist supervision.

Clinical trial registration no.: NCT03753945 (ClinicalTrials.gov).

https://thejns.org/doi/abs/10.3171/2019.12.SPINE191241

KEYWORDS magnetic resonance imaging; spine; safety; implants; deep brain stimulation; neurostimulator; functional neurosurgery; diagnostic technique

$\mathrm{T}$ o date, more than 150,000 patients have received deep brain stimulation (DBS) neuromodulation devices worldwide. ${ }^{14}$ DBS is best established as a therapy for movement disorders, including Parkinson's disease (PD), essential tremor, and dystonia. In recent years, it has also become of interest as a treatment strategy for psychiatric and cognitive disorders, such as major depressive disorder, obsessive-compulsive disorder, and, possibly, Alzheimer's disease. ${ }^{21}$ DBS surgery entails the insertion of electrodes into deep brain structures and mod-

ABBREVIATIONS B1+rms = root-mean-square value of the MRI effective component of the radiofrequency magnetic $\left(B_{1}\right)$ field; $C$-spine $=$ cervical spine; $D B S=$ deep brain stimulation; IPG = implantable pulse generator; L-spine = lumbar spine; PD = Parkinson's disease; RF = radiofrequency; $S A R=$ specific absorption rate; T-spine = thoracic spine; T1W = T1-weighted; T2W = T2-weighted

SUBMITTED October 16, 2019. ACCEPTED December 6, 2019.

INCLUDE WHEN CITING Published online February 14, 2020; DOI: 10.3171/2019.12.SPINE191241. 
ulation of desired brain targets using electric current. DBS electrodes are connected to an implantable pulse generator (IPG) via extension wires that are tunneled under the skin. The IPG is typically placed subcutaneously in the upper chest wall. Because of safety concerns and stringent vendor guidelines, MRI of patients following DBS implantation is highly restricted. ${ }^{7,12,20,33}$ Prior studies have investigated the safety of brain MRI in these patients, ${ }^{6,7}$, 13,26 but safety data for MRI of other body parts, such as the spine, are lacking.

Due to 3 previous cases of DBS patient injuries presumed to be related to MRI, MRI guidelines for DBS systems have become restrictive, as well as vendor and hardware specific. ${ }^{15,31,35}$ In particular, one case of perielectrode hemorrhage and permanent neurological changes occurred during a 1.0-T lumbar spine (L-spine) MRI session. ${ }^{15}$ At present, FDA-approved MRI conditional DBS devices are available from Medtronic, Boston Scientific, and Abbott. ${ }^{5,23,32}$ Depending on the models of the DBS leads, extension wires, and IPG, patients may be eligible for head or full-body MRI. For MRI safety purposes, the spine is considered within the category of "full body." DBS devices are approved for MRI at specific magnetic field strengths (i.e., 1.5 T) and with specific heating-related thresholds. These thresholds are based on a specific absorption rate (SAR) and root-mean-square (rms) value of the MRI effective component of the radiofrequency (RF) magnetic $\left(\mathrm{B}_{1}\right)$ field $(\mathrm{B} 1+\mathrm{rms})$. The SAR should remain $\leq 0.1 \mathrm{~W} / \mathrm{kg}$ during the MRI session. ${ }^{5,23}$ These MRI restrictions are in place mainly to prevent heating of the DBS system (particularly the electrode tips) and potential brain injury. ${ }^{6}{ }^{13}$ However, these guidelines prohibit the use of most routine clinical MRI protocols, unless they are modified. SAR or B1+rms requirements can be met by reducing the number of slices/echoes or increasing the repetition time, which usually lengthens the acquisition time. In addition, reducing the number of slices typically warrants radiologist supervision to ensure that adequate anatomy is imaged. ${ }^{1}$

With the exception of acute intracranial indications, MRI is the diagnostic imaging modality of choice for most cranial and spinal clinical queries. CT of the spine is insufficient for visualizing nonbony structures, while CT myelography is generally inferior to MRI and can be associated with complications such as bleeding, infection, seizures, and contrast-induced nephrotoxicity. ${ }^{24,27}$ Underscoring the clinical demand for MRI in this unique patient population, one study estimated that $66 \%-75 \%$ of patients diagnosed with PD, essential tremor, or dystonia need to undergo MRI within 10 years of DBS surgery. ${ }^{10}$ With most of these indications (62\%) calling for body and/or spine MRI, there is evident value in establishing a safe MRI protocol for these patients..$^{10}$ Except for a single study in the early 2000s comparing brain and chest MRI safety, ${ }^{19}$ to our knowledge, no studies have investigated the safety profile of body MRI, particularly spine MRI, in DBS patients.

Similar to our previous safety studies for brain MRI, ${ }^{6,13,26}$ the goals of this study were to: 1 ) investigate the safety of spine MRI outside the prescribed vendor guidelines using a phantom model, 2) thereby establish safe protocols for spine MR image acquisition with optimized acquisition parameters (e.g., the number of slices), and 3) validate these protocols clinically by prospectively imaging a cohort of DBS patients who required spine MRI.

\section{Methods \\ Phantom Safety Data}

To evaluate heating of the DBS system during MRI, a phantom study was conducted. The experimental setup has recently been described by our group., ${ }^{6,13}$ Briefly, a Lucite phantom was equipped with a full-body, eligible DBS device consisting of two DBS leads (model 3387; Medtronic), two extension wires (model 37086, $60 \mathrm{~cm}$; Medtronic), and an IPG (Activa PC model 37601; Medtronic). In this phantom experiment, we did not test IPG output stability; the IPG was turned off during scanning. This was done because patients would undergo spine MRI either without stimulation (if tolerated) or with bipolar stimulation, which is approved by Medtronic guidelines. ${ }^{23}$

The phantom was placed head first and in supine position in the MRI scanner isocenter and was landmarked at the typical location for cervical (C-), thoracic (T-), or L-spine imaging to simulate conventional patient positioning. All experiments were performed with a 1.5-T MRI (GE Signa Excite MRI, software level HD 12x; GE Healthcare). A transmit-body spine-receive coil (8-channel coil, GE model 5268673) was used on the phantom, corresponding to the $\mathrm{C}$-spine, T-spine, and $\mathrm{L}$-spine. In contrast to head MRI, where a single RF coil is usually used to transmit the RF and receive the signal, spine MRI utilizes a transmit coil that is integrated into the bore of the scanner. This transmit-body coil exposes the DBS system to higher RF and can generate more device heating. The pulse sequences tested are listed in Table 1.

Similar to recent investigations by our group,${ }^{6,13}$ temperature changes were measured at the most distal contacts of each lead using two fiber-optic temperature probes connected to a fiber-optic thermometer (model 790; Luxtron; $0.1^{\circ} \mathrm{C}$ temperature resolution). Additionally, a third thermometer (model FTI-10; FISO; $0.01^{\circ} \mathrm{C}$ temperature resolution) was placed adjacent to the posterior aspect of the IPG. Temperature change was defined as the difference between the baseline temperature, as measured prior to sequence initiation, and the so-called during-sequence temperature (including pre-scans). Once the recorded temperature had remained stable for a duration of at least 1 minute, during-sequence temperature rises were computed by averaging the temperature of the last 5 seconds recorded. This was done because the highest temperature would be expected with longer scanning time (logarithmic increase in temperature over time ${ }^{3,4}$ ) and because the fiber-optic probes' high sensitivity creates slight variations across data points.

We first investigated the temperature rises associated with cervical, thoracic, and lumbar protocols that are routinely employed for spine imaging at our institution. Once a pulse sequence triggered a temperature rise $>2{ }^{\circ} \mathrm{C}$, it was deemed unsafe, and scanning was aborted. In such cases, protocols were adjusted in a stepwise manner to decrease heating: first, if the employed protocol entailed 
TABLE 1. Summary of pulse sequences and associated parameters used for the various experiments

\begin{tabular}{|c|c|c|c|c|c|c|c|c|c|c|c|c|c|c|}
\hline $\begin{array}{l}\text { Spine } \\
\text { Image }\end{array}$ & Pulse Sequence & $\begin{array}{c}\text { TR } \\
\text { (msec) }\end{array}$ & $\begin{array}{c}\mathrm{TE} \\
(\mathrm{msec})\end{array}$ & $\begin{array}{c}\mathrm{TI} \\
\text { (msec) }\end{array}$ & $\begin{array}{c}\text { BW } \\
(\mathrm{kHz})\end{array}$ & $\begin{array}{l}\text { FOV } \\
(\mathrm{mm})\end{array}$ & $\mathrm{FA}\left({ }^{\circ}\right)$ & $\begin{array}{l}\text { No. of } \\
\text { Slices }\end{array}$ & $\begin{array}{c}\mathrm{ST} \\
(\mathrm{mm})\end{array}$ & $\begin{array}{l}\text { Gap } \\
(\mathrm{mm})\end{array}$ & ETL & Matrix & $\begin{array}{c}\text { Frequency-Encoding } \\
\text { Direction }\end{array}$ & NEX \\
\hline $\mathrm{C} / \mathrm{T}$ & 3-plane Loc & 5.2 & 1.6 & - & 31.25 & 48 & 80 & - & 5.0 & 10.0 & - & $256 \times 128$ & $\mathrm{Rt} / \mathrm{tt}$ & 1.00 \\
\hline $\mathrm{T} / \mathrm{L}$ & 3-plane Loc & 5.2 & 1.6 & - & 31.25 & 48 & 80 & - & 5.0 & 10.0 & - & $256 \times 128$ & $\mathrm{Rt} / \mathrm{t}$ & 1.00 \\
\hline C & Cal scan & 150 & 1.4 & - & 31.2 & 48 & 70 & - & 9.0 & 0 & - & $32 \times 32$ & $\mathrm{Rt} / \mathrm{t}$ & 1.00 \\
\hline C & 3D Ax FRFSE T2W* & 2200 & 102.0 & - & 41.67 & 18.0 & $\mathrm{XL} / 90$ & - & 2.5 & 0 & 39 & $320 \times 224$ & $\mathrm{Rt} / \mathrm{t}$ & 1.00 \\
\hline C & Ax T2W 2D \#1 & 2500 & 85.0 & - & 25.00 & 20 & $\mathrm{XL} / 90$ & 8 & 4.0 & 0.5 & 15 & $256 \times 224$ & $\mathrm{~A} / \mathrm{P}$ & 2.00 \\
\hline C & Ax T2W 2D \#2 & 3000 & 85 & - & 25.00 & 20 & $\mathrm{XL} / 90$ & 8 & 4.0 & 0.5 & 8 & $256 \times 224$ & $A / P$ & 2.00 \\
\hline C & Sag T1W* & 450 & Min & - & 25.0 & 22.0 & SE & 13 & 3.0 & 0.5 & - & $384 \times 256$ & $A / P$ & 3.00 \\
\hline C & Sag T1W \#1 & 500 & Min & - & 25.00 & 22 & SE & 3 & 3.0 & 0.5 & - & $384 \times 256$ & $A / P$ & 3.00 \\
\hline C & Sag FRFSE T2W* & 5800 & 114.0 & - & 41.67 & 22.0 & $\mathrm{XL} / 90$ & 13 & 3.0 & 0.5 & 33 & $384 \times 256$ & $\mathrm{~A} / \mathrm{P}$ & 4.00 \\
\hline C & Sag FRFSE T2W \#1 & 3000 & 114.0 & - & 41.67 & 22.0 & $\mathrm{XL} / 90$ & 6 & 3.0 & 0.5 & 33 & $384 \times 256$ & $\mathrm{~A} / \mathrm{P}$ & 4.00 \\
\hline C & Sag FRFSE T2W \#2 & 3000 & 114.0 & - & 41.67 & 22.0 & $\mathrm{XL} / 90$ & 3 & 3.0 & 0.5 & 33 & $384 \times 256$ & $A / P$ & 4.00 \\
\hline $\mathrm{T} / \mathrm{L}$ & Cal scan & 150 & 1.4 & - & 31.2 & 48 & 70 & - & 9.0 & 0 & - & $32 \times 32$ & $\mathrm{Rt} / \mathrm{lt}$ & 1.00 \\
\hline $\mathrm{T}$ & Ax 3D FRFSE* & 2200 & 102 & - & 41.67 & 18.0 & $\mathrm{XL} / 90$ & - & 2.5 & 0 & 39 & $320 \times 224$ & $\mathrm{Rt} / \mathrm{t}$ & 1.00 \\
\hline$T$ & Ax FRFSE 2D \#1 & 3000 & 85.0 & - & 25.00 & 20.0 & $\mathrm{XL} / 90$ & 20 & 4.0 & 0.5 & 15 & $256 \times 224$ & $A / P$ & 2.00 \\
\hline$T$ & Ax T2W 2D \#2 & 4000 & 85.0 & - & 25.00 & 20 & $\mathrm{XL} / 90$ & 20 & 4.0 & 0.5 & 15 & $256 \times 124$ & $A / P$ & 2.00 \\
\hline $\mathrm{T}$ & Sag T1W* & 500 & Min & - & 15.63 & 32.0 & SE & 15 & 3.0 & 0.5 & - & $320 \times 256$ & $\mathrm{~A} / \mathrm{P}$ & 3.00 \\
\hline $\mathrm{T}$ & Sag T1W \#1 & 400 & Min & - & 15.63 & 32.0 & SE & 6 & 3.0 & 0.5 & - & $256 \times 256$ & $A / P$ & 3.00 \\
\hline $\mathrm{T}$ & Sag FRFSE T2W* & 4350 & 116.0 & - & 41.67 & 32.0 & $\mathrm{XL} / 90$ & 17 & 3.0 & 0.5 & 27 & $320 \times 256$ & $A / P$ & 4.00 \\
\hline$T$ & Sag FRFSE T2W \#1 & 4350 & 116 & - & 41.67 & 32.0 & $\mathrm{XL} / 90$ & 8 & 3.0 & 0.5 & 27 & $320 \times 256$ & $A / P$ & 4.00 \\
\hline$T / L$ & Cal scan & 150 & 1.4 & - & 31.2 & 48 & 70 & - & 9.0 & 0 & - & $32 \times 32$ & $\mathrm{Rt} / \mathrm{t}$ & 1.00 \\
\hline L & Ax T2W 2D* & 4800 & 85.0 & - & 25.00 & 20.0 & $\mathrm{XL} / 90$ & 23 & 4.0 & 0.5 & 15 & $256 \times 224$ & $A / P$ & 2.00 \\
\hline L & Sag T1W* & 350 & Min & - & 31.25 & 32 & SE & 9 & 3.0 & 0.5 & - & $384 \times 256$ & $\mathrm{Rt} / \mathrm{lt}$ & 2.00 \\
\hline L & Sag FRFSE T2W* & 4000 & 107.0 & - & 25.00 & 32 & $\mathrm{XL} / 90$ & 14 & 3.0 & 0.5 & 19 & $384 \times 256$ & Rt/lt & 4.00 \\
\hline
\end{tabular}

$\mathrm{A}=$ anterior; $\mathrm{Ax}=$ axial; $\mathrm{BW}=$ bandwidth; $\mathrm{Cal}=$ calibration; $\mathrm{ETL}=$ echo train length; FA = flip angle; FOV = field of view; FRFSE = fast relaxation fast spin echo se-

quence; Loc = localizer; Min = minimum; NEX = number of excitations; $\mathrm{P}=$ posterior; Sag = sagittal; $\mathrm{SE}=$ spin echo; $\mathrm{ST}=$ slice thickness; TE = echo time; $\mathrm{TI}=$ inversion time; $\mathrm{TR}=$ repetition time; $\mathrm{XL}=$ accelerated $;-=$ not applicable.

Sequences routinely used at our institution are identified with an asterisk $\left({ }^{*}\right)$, and modified sequences deemed to be safe $\left(<2^{\circ} \mathrm{C}\right)$ during the phantom study are shown in boldface. To ensure patient safety during imaging, 3 additional safety measures were taken: 1) routinely performed calibration scans were omitted, 2) the overall number of slices in each sequence was reduced by 33\% (e.g., T-spine sagittal T1W \#1 [i.e., T-spine Sag T1W \#1] would become 4 slices when scanning patients), and 3) T-spine sequences were used for imaging of both the T- and L-spine, since the lack of a detectable temperature rise during L-spine sequences precluded the establishment of a safety threshold for these sequences (refer to Table 3).

a $3 \mathrm{D}$ scan, the sequence was changed from $3 \mathrm{D}$ to $2 \mathrm{D}$; second, the number of slices was gradually reduced until temperature changes were within acceptable limits $(<$ $2^{\circ} \mathrm{C}$ ); and third, repetition-time lengthening was similarly performed but to a lesser extent. Probes were inspected immediately after scanning to ensure that no movement had occurred.

In addition to absolute temperature differences, the SAR - a parameter associated with implant heating - was also measured. Whole-body SAR values, assuming a patient weight of $70 \mathrm{~kg}$, were recorded from the scanner console during imaging. The SAR is the dosimetric standard to characterize the thermogenic aspects of an electromagnetic field. To determine the relationship between temperature rise and the SAR, each temperature rise was divided by the associated SAR, yielding a $\Delta \mathrm{T} / \mathrm{SAR}$ value.

\section{Study Participants}

Following ethics approval (University Health Network Research Ethics Board), and for the prospective component of this study (clinical trial registration no.: NCT03753945, clinicaltrials.gov), we recruited participants who required a spine MRI study as per assessment by their treating physicians (Fig. 1). All patients provided written informed consent. Inclusion criteria were as follows: 1) participants receiving active DBS system at any therapeutic targets, 2) ability to provide written informed consent, and 3) implantation of specific models of Medtronic DBS hardware (leads: 3387 or 3389; extension wire: 37086, $60 \mathrm{~cm}$; IPG: Activa PC 37601, Activa RC 37612, or Activa SC 37603) with a configuration similar to that tested in the phantom experiments. DBS device models with comparable MRI safety guidelines to those used in the phantoms were deemed acceptable. Participants who were unable to give written informed consent (e.g., owing to cognitive or psychological impairment) or who required nonroutine spine protocols (e.g., using a contrast agent) were excluded. Patients with electrode impedances outside vendor guidelines (monopolar $>2000 \Omega$ and bipolar $<250 \Omega$ ) ) $^{23}$ and clinical indication of a short or open circuit (e.g., recent 
Referred patients

$(\mathrm{n}=18)$

Active DBS at any target

Clinically required spine MRI

Excluded

- Unable to give informed consent $(n=0)$

- DBS hardware not tested in phantom studies $(\mathrm{n}=2)$

- Indications requiring contrast $(n=5)$

- Nonroutine spine MRI protocols $(\mathrm{n}=2)$

Eligible patient

$(\mathrm{n}=9)$

Unable to acquire MRI

- Claustrophobia/Anxiety $(\mathrm{n}=0)$

- Excessive movement at rest $(n=0)$

Eligible for MRI scan $(\mathrm{n}=9)$<smiles>[3H][CH]</smiles>

No. of acquired sequences (refer to Table 5)

FIG. 1. Study flowchart showing participant recruitment and referrals from treating physicians and inclusion/exclusion criteria.

change in clinical efficacy or sudden impedance changes at the previous DBS clinic visit) were also excluded. Study protocols and data generated during the study are available from the corresponding author upon request.

From March 2019 to early July 2019, we performed 1.5-T MRI (GE Signa Excite MRI, software level HD 12×; GE Healthcare) with a transmit-body spine-receive coil (8-channel coil, GE model 5268673) in 9 participants with fully internalized DBS systems (Table 2). If tolerated by participants, MR images were acquired with the DBS device turned off; otherwise, the DBS was left on in a bipolar stimulation configuration for the duration of the MRI session in accordance with current vendor guidelines. ${ }^{23}$ Patients in whom monopolar stimulation settings were used were temporarily put on bipolar settings of approximate clinical equivalency according to a specific algorithm (i.e., the cathode remained unchanged, an adjacent contact was set to be the anode, and the voltage was increased by $20 \%-30 \%) .{ }^{9,25}$ To ensure safety during imaging, only pulse sequences that yielded temperature increases $<2^{\circ} \mathrm{C}$ in the phantom experiment (listed in bold in Table 1) were employed in participants. Furthermore, routinely performed calibration scans were omitted, and the overall number of slices in each sequence was reduced by $33 \%$. Finally, T-spine sequences were used for imaging of both the T- and the L-spine, since the lack of detectable temperature rise during L-spine sequences precluded the establishment of a safety threshold for these sequences (Table 3). In the event that the number of slices in a given sequence provided insufficient anatomical coverage, the
TABLE 2. Summary of DBS participants' demographic and clinical data $(n=9)$

\begin{tabular}{lc}
\hline \multicolumn{1}{c}{ Demographic \& Clinical Data } & Value \\
\hline Age (yrs), mean & $62.4 \pm 12.3$ \\
\hline Sex & $2(22)$ \\
\hline Female & $7(78)$ \\
\hline Male & $39.9 \pm 23.2$ \\
\hline Time btwn MRI \& DBS surgery (mos), mean & $5(56)$ \\
\hline No. of patients w/ DBS-on during MRI & 7 \\
\hline Primary DBS indication/target & 1 \\
\hline PD/subthalamic nucleus & 1 \\
\hline PD/globus pallidus interna & \\
\hline Dystonia/globus pallidus interna
\end{tabular}

Unless otherwise specified, values are presented as the number of participants (\%). Mean values are presented \pm SD.

sequence was repeated, visualizing the contiguous anatomy. Between each sequence, scanning was held for 45 seconds to allow for heat dissipation (Fig. 2). Finally, a T2weighted (T2W) brain MRI sequence (3-plane localizer and axial T2W image: TR $4000 \mathrm{msec}$, TE $85 \mathrm{msec}, 0.5$ $\times 0.5 \times 3-\mathrm{mm}$ voxels) covering the contact portion of the DBS leads was performed before and after the spine MRI to assess for acute intracranial findings such as edema. This T2W brain MRI study was obtained using a transmit-receive head coil (GE model 2341973), consistent with vendor guidelines.

The primary outcome measure of this prospective trial was short-term adverse events as detected during a complete neurological examination immediately after MRI. Motor, sensory (upper and lower limbs), and cranial nerve functions were evaluated, as were alertness and orientation. Any clinical changes from baseline were considered an adverse event. Secondary outcomes included the following: 1) long-term adverse effects assessed at latest routine DBS visits after MRI (any clinical change from baseline was considered an adverse event); 2) acute intracranial findings on immediate post-imaging brain MRI (defined as signal changes adjacent to the neurostimulator other than the typical metallic artifact); and 3) change in electrical circuit integrity of the DBS system as evaluated by comparison of pre- and post-MRI DBS impedances (Activa Patient Programmer; 37642; Medtronic).

For each patient, whole-body SAR values were recorded from the scanner console.

\section{Statistical Analysis}

For the phantom, analysis of temperature rises and SARs was conducted using $\mathrm{R}$ (version 3.4.3, https:// www.r-project.org/).

For the study participants, a paired t-test was used to assess the difference in impedances before and after spine MRI. Any $p$ value $<0.05$ was considered statistically significant. Statistical analyses were performed using R (version 3.4.3, https://www.r-project.org/). Mean values are presented $\pm \mathrm{SD}$. 
TABLE 3. Pulse sequences and associated parameters used in patients

\begin{tabular}{|c|c|c|c|c|c|c|c|c|c|c|c|c|c|c|}
\hline $\begin{array}{l}\text { Spine } \\
\text { Region }\end{array}$ & Pulse Sequence & $\begin{array}{c}\text { TR } \\
\text { (msec) }\end{array}$ & $\begin{array}{c}\mathrm{TE} \\
\text { (msec) }\end{array}$ & $\begin{array}{c}\mathrm{TI} \\
\text { (msec) }\end{array}$ & $\begin{array}{c}\text { BW } \\
(\mathrm{kHz})\end{array}$ & $\begin{array}{l}\text { FOV } \\
(\mathrm{mm})\end{array}$ & $\mathrm{FA}\left({ }^{\circ}\right)$ & $\begin{array}{l}\text { No. of } \\
\text { Slices }\end{array}$ & $\begin{array}{l}\mathrm{ST} \\
(\mathrm{mm})\end{array}$ & $\begin{array}{l}\text { Gap } \\
(\mathrm{mm})\end{array}$ & ETL & Matrix & $\begin{array}{l}\text { Frequency-Encoding } \\
\text { Direction }\end{array}$ & NEX \\
\hline$C \& T$ & 3-plane Loc & 5.2 & 1.6 & 600 & 31.25 & 48 & 80 & - & 5.0 & 10.0 & - & $256 \times 128$ & $\mathrm{Rt} / \mathrm{lt}$ & 1.00 \\
\hline$T \& L$ & 3-plane Loc & 5.2 & 1.6 & 600 & 31.25 & 48 & 80 & - & 5.0 & 10.0 & - & $256 \times 128$ & $\mathrm{Rt} / \mathrm{t}$ & 1.00 \\
\hline C & $\mathrm{Ax}$ T2W 2D \#2 & 3000 & 85 & - & 25.00 & 20 & $\mathrm{XL} / 90$ & 5 & 4.0 & 0.5 & 8 & $256 \times 224$ & $A / P$ & 2.00 \\
\hline C & Sag T1W \#1 & 500 & Min & - & 25.00 & 22 & SE & 2 & 3.0 & 0.5 & - & $384 \times 256$ & $A / P$ & 3.00 \\
\hline C & Sag FRFSE T2W \#2 & 3000 & 114.0 & - & 41.67 & 22.0 & $\mathrm{XL} / 90$ & 2 & 3.0 & 0.5 & 33 & $384 \times 256$ & $A / P$ & 4.00 \\
\hline$T \& L$ & $\mathrm{Ax}$ T2W 2D \#2 & 4000 & 85.0 & - & 25.00 & 20 & $\mathrm{XL} / 90$ & 13 & 4.0 & 0.5 & 15 & $256 \times 124$ & $A / P$ & 2.00 \\
\hline$T \& L$ & Sag T1W \#1 & 400 & Min & - & 15.63 & 32.0 & SE & 4 & 3.0 & 0.5 & - & $256 \times 256$ & $A / P$ & 3.00 \\
\hline$T \& L$ & Sag FRFSE T2W \#1 & 4350 & 116 & - & 41.67 & 32.0 & $\mathrm{XL} / 90$ & 5 & 3.0 & 0.5 & 27 & $320 \times 256$ & $\mathrm{~A} / \mathrm{P}$ & 4.00 \\
\hline
\end{tabular}

The parameters are based on modified sequences deemed to be safe $\left(<2^{\circ} \mathrm{C}\right)$ during the phantom study.

\section{Results}

\section{Phantom Data Suggest Safe Imaging Outside of Prescribed Vendor Guidelines}

Table 4 summarizes the observed temperature increases at the electrode tips. Except for the localizer of the upper spine, temperature rises were higher at the right electrode tip compared to the left electrode tip. The thermometer probe adjacent to the posterior surface of the IPG detected no changes in temperature across all employed sequences.

A craniocaudal temperature gradient was observed along the different levels of the spine during imaging (Table 4). The routine L-spine protocol sequences triggered a maximum temperature rise of $0.2^{\circ} \mathrm{C}$, whereas all routine sequences used for the $\mathrm{C}$ - and $\mathrm{T}$-spine were associated with at least a $1.7^{\circ} \mathrm{C}$ temperature increase (Fig. $3)$. By changing $3 \mathrm{D}$ to $2 \mathrm{D}$ acquisition and reducing the number of slices-up to $77 \%$ and $60 \%$ for the C-spine and T-spine, respectively-we were able to decrease heating at the electrode tips to $<2{ }^{\circ} \mathrm{C}$ for all sequences, thereby establishing a safe protocol to use for participants. The dynamics of scanning-related temperature rises are shown in an exemplar sequence (i.e., routine axial T2W C-spine sequence) as well as the modified and safe version of the sequence in Fig. 2.

Notably, all estimated whole-body SAR values were outside prescribed vendor guidelines $(>0.1 \mathrm{~W} / \mathrm{kg})$, including the sequences deemed safe. SARs varied from $0.3 \mathrm{~W} / \mathrm{kg}$ (C-spine sagittal T1-weighted [T1W] image) to $1.8 \mathrm{~W} / \mathrm{kg}$ (localizer upper spine). The calculated $\Delta \mathrm{T} / \mathrm{SAR}$ was highest for the $\mathrm{C}$-spine $\left(2.3^{\circ} \mathrm{C} /[\mathrm{W} / \mathrm{kg}]\right)$, followed by the T-spine $\left(1.1^{\circ} \mathrm{C} /[\mathrm{W} / \mathrm{kg}]\right)$, and reached negligible values during $\mathrm{L}$-spine sequences $\left(0.1^{\circ} \mathrm{C} /[\mathrm{W} / \mathrm{kg}]\right)$. Figure 4 features a linear model displaying the relationship between temperature increase and the SAR. Based on this relationship, SAR thresholds associated with a temperature rise of $2^{\circ} \mathrm{C}$ were estimated for each spine segment. The maximum employable SAR within safe limits was lowest for the C-spine $(0.9 \mathrm{~W} / \mathrm{kg})$ and $\mathrm{T}$-spine $(1.8 \mathrm{~W} / \mathrm{kg})$ and
A

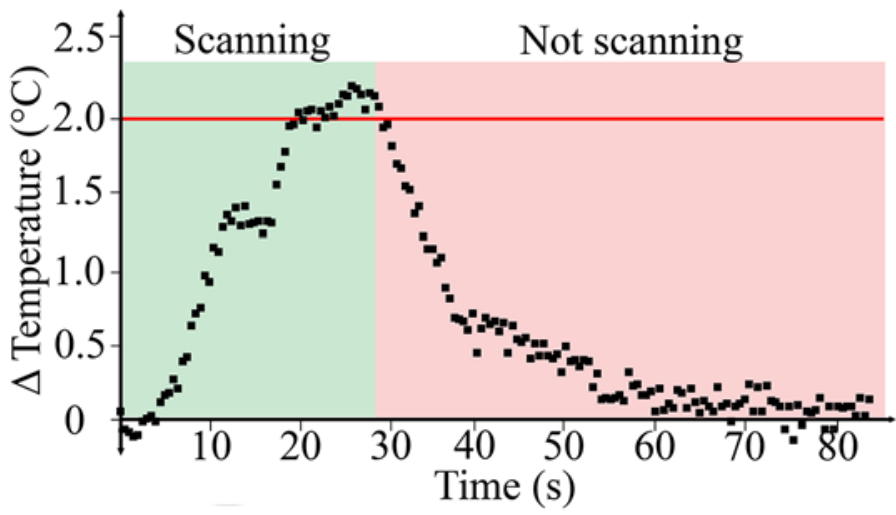

B

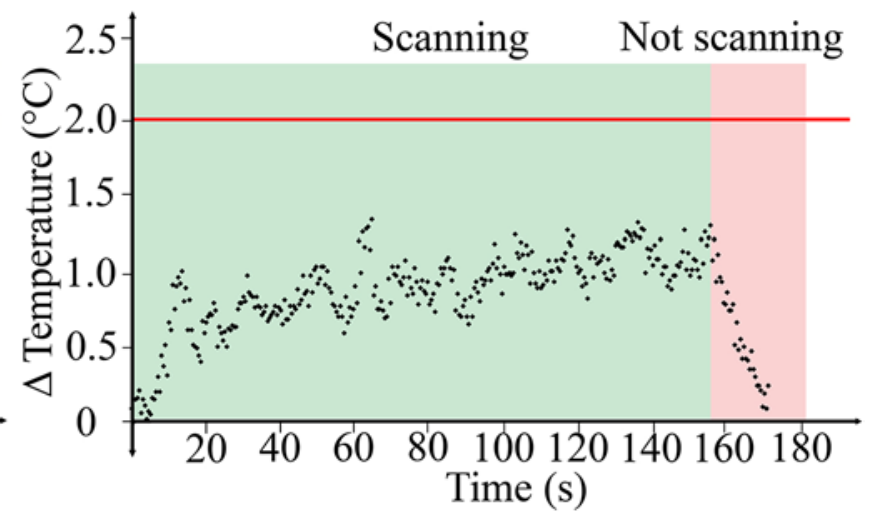

FIG. 2. Dynamics of temperature rise in a phantom model. A: Representation of temperature rises over time in an unsafe spine sequence (routinely used $\mathrm{C}$-spine axial T2W image). Within 20 seconds of scanning initiation, the temperature increased rapidly above $2^{\circ} \mathrm{C}$. As scanning was discontinued, the temperature exhibited an exponential decline, returning to baseline after approximately 45 seconds. B: Modified axial C-spine T2W imaging with a temperature of $<2^{\circ} \mathrm{C}$ is deemed safe. The routine axial $\mathrm{C}$-spine T2W image was transformed into a $2 \mathrm{D}$ sequence with slightly longer repetition time. The red line indicates a safe threshold of $2^{\circ} \mathrm{C}$. Figure is available in color online only. 
TABLE 4. Temperature rises measured at the electrode tips

\begin{tabular}{|c|c|c|c|c|}
\hline \multirow{2}{*}{$\begin{array}{c}\text { Region/Pulse } \\
\text { Sequence }\end{array}$} & \multicolumn{3}{|c|}{ Temperature Measurements $\left({ }^{\circ} \mathrm{C}\right)$} & \multirow{2}{*}{$\begin{array}{c}\text { SAR } \\
(\mathrm{W} / \mathrm{kg})\end{array}$} \\
\hline & Right & Left & IPG & \\
\hline \multicolumn{5}{|l|}{ Cervical spine } \\
\hline Localizer top & 1.0 & 1.4 & 0 & 1.8 \\
\hline Localizer bottom & 0.2 & 0.1 & 0 & 1.8 \\
\hline Calibration & $>2$ & 0 & 0 & 1.6 \\
\hline $\mathrm{Ax} \mathrm{T}_{2} \mathrm{~W}^{*}$ & $>2$ & 0 & 0 & 1 \\
\hline Ax T2W \#1 & 1.9 & 0 & 0 & 0.5 \\
\hline Ax T2W \#2 & 1.2 & 0.2 & 0 & 0.4 \\
\hline Sag T1W* & $>2$ & 0 & 0 & 1.7 \\
\hline Sag T1W \#1 & 1.1 & 0.1 & 0 & 0.3 \\
\hline Sag T2W* & $>2$ & 0 & 0 & 0.9 \\
\hline Sag T2W \#1 & 1.7 & 0.1 & 0 & 0.8 \\
\hline Sag T2W \#2 & 1.2 & 0.2 & 0 & 0.4 \\
\hline \multicolumn{5}{|l|}{ Thoracic spine } \\
\hline Calibration & 0.9 & 0 & 0 & 1.6 \\
\hline $\mathrm{Ax} \mathrm{T}^{2} \mathrm{~W}^{*}$ & $>2$ & 0.4 & 0 & 1.3 \\
\hline $\mathrm{Ax}$ T2W \#1 & 1.8 & 0.3 & 0 & 1 \\
\hline Ax T2W \#2 & 0.8 & 0.2 & 0 & 0.6 \\
\hline Sag T1W* & 1.9 & 0.8 & 0 & 1.7 \\
\hline Sag T1W \#1 & 1 & 0 & 0 & 0.9 \\
\hline Sag T2W* & 1.7 & 0.5 & 0 & 1.3 \\
\hline Sag T2W \#1 & 0.7 & 0 & 0 & 0.6 \\
\hline \multicolumn{5}{|l|}{ Lumbar spine } \\
\hline Calibration & 0.1 & 0.1 & 0 & 1.4 \\
\hline Ax T2W* & 0 & 0 & 0 & 1 \\
\hline Sag T1W* & 0.1 & 0.1 & 0 & 1.5 \\
\hline Sag T2W* & 0.2 & 0 & 0 & 0.9 \\
\hline
\end{tabular}

The rises in temperature measured during various experimental configurations using MRI pulse sequences are shown in the "Right" and "Left" columns, measuring the rises in temperature at the right and left electrodes, respectively. The estimated whole-body SAR is also shown. Sequences routinely used at our institution are identified with an asterisk (*), and modified sequences deemed to be safe $\left(<2^{\circ} \mathrm{C}\right)$ during the phantom study are shown in boldface.

reached very high values during imaging of the L-spine $(25.6 \mathrm{~W} / \mathrm{kg})$.

\section{Validation of Phantom Safety Data in Participants With DBS Devices}

Safety protocols derived from the phantom study (Table 3 ) were clinically validated in a cohort of 9 patients (67 individual spine sequences overall; Table 5) whose mean age was $62.4 \pm 12.3$ years (Table 2); 7 participants were male and 2 were female. Exemplar spine images are shown in Fig. 5. There were no acute MRI-related short- or longterm adverse events (mean follow-up period $91 \pm 57$ days). Limited T2W brain MRI demonstrated no acute brain changes following spine MRI.

To cover the entire anatomy needed to answer the clinical question, sequences may have been repeated in a spatially contiguous manner. The mean number of spatially contiguous acquisitions per patient was 2 (range 1-3) for axial T2W images (C-, T-, and L-spine), 2 (range 1-3) for sagittal T1W images (C-, T-, and L-spine), and 3 (range 1-5) for sagittal T2W images (C-, T-, and L-spine) (Table 5). Similar to routine spine protocols, sagittal T2W images typically included full anatomical spine coverage, whereas other sequences, such as axial T2W images, were limited to the abnormal spine levels and may thus have had variable acquisition times. To compare acquisition time of our phantom-based safe protocol to routine clinical protocols, we therefore only compared sagittal T2W sequences, which had full spine anatomical coverage in both cases (Table 6). For the phantom-based protocol, the mean sagittal C- and L-spine sagittal T2W acquisition times were 8.5 minutes (single patient) and $10.8 \pm 2.7$ minutes (multiple patients), respectively. For routine protocols, the mean sagittal C- and L-spine sagittal T2W acquisition times were 3.2 and 4.6 minutes, respectively.

Prior to MRI, the mean DBS system impedance was $1602.9 \pm 817.6 \Omega$. After MRI, the mean DBS system impedance was $1608.6 \pm 819.7 \Omega$ (mean of changes $5.7 \pm$ $117.0 ;>0.1$, paired t-test). No significant differences were found between pre- and post-MRI impedances.

\section{SAR Estimations in Participants With DBS}

Mean SARs were $0.29 \mathrm{~W} / \mathrm{kg}$ (range 0.26-0.32 W/kg) for the C-spine, $0.46 \mathrm{~W} / \mathrm{kg}$ (range $0.44-0.47 \mathrm{~W} / \mathrm{kg}$ ) for the T-spine, and $0.64 \mathrm{~W} / \mathrm{kg}$ (range $0.32-1.06 \mathrm{~W} / \mathrm{kg}$ ) for the Lspine. Similar to the phantom data, these values were all outside the vendor recommended guidelines $(>0.1 \mathrm{~W} / \mathrm{kg})$.

\section{Discussion}

The present study established safe spine MRI protocols in a DBS phantom model and successfully validated these protocols in a cohort of 9 DBS patients (67 spine imaging sequences) with fully internalized DBS systems who clinically required MRI of the C-, T-, or L-spine. A suboptimal alternative would have been a spine CT scan. To achieve safe spine imaging, the number of slices acquired per sequence was reduced, and sequences were repeated in a spatially contiguous fashion to provide the necessary anatomical coverage. Notably, this established protocol used a higher SAR than recommended, which allowed maximization of the number of slices acquired per sequence compared to the number of slices that would have been acquired with protocols respecting guidelines. The lack of both short- and long-term adverse effects and the absence of acute intracranial findings on T2W brain imaging following spine imaging indicate the safety of the protocols employed. Finally, we demonstrated a steep safety gradient along the length of the spine: L-spine scanning triggered no appreciable rise in temperature, whereas routinely used $\mathrm{C}$-spine MRI protocols produced unacceptable heating. To our knowledge, this is the first MRI safety study to investigate body MRI in patients with DBS.

Recently, specific models have been deemed full-body MRI eligible. This change in guidelines will dramatically impact the care for patients with DBS, given that nearly $75 \%$ of patients are expected to require MRI within 10 years of DBS surgery, with more than half of the indica- 


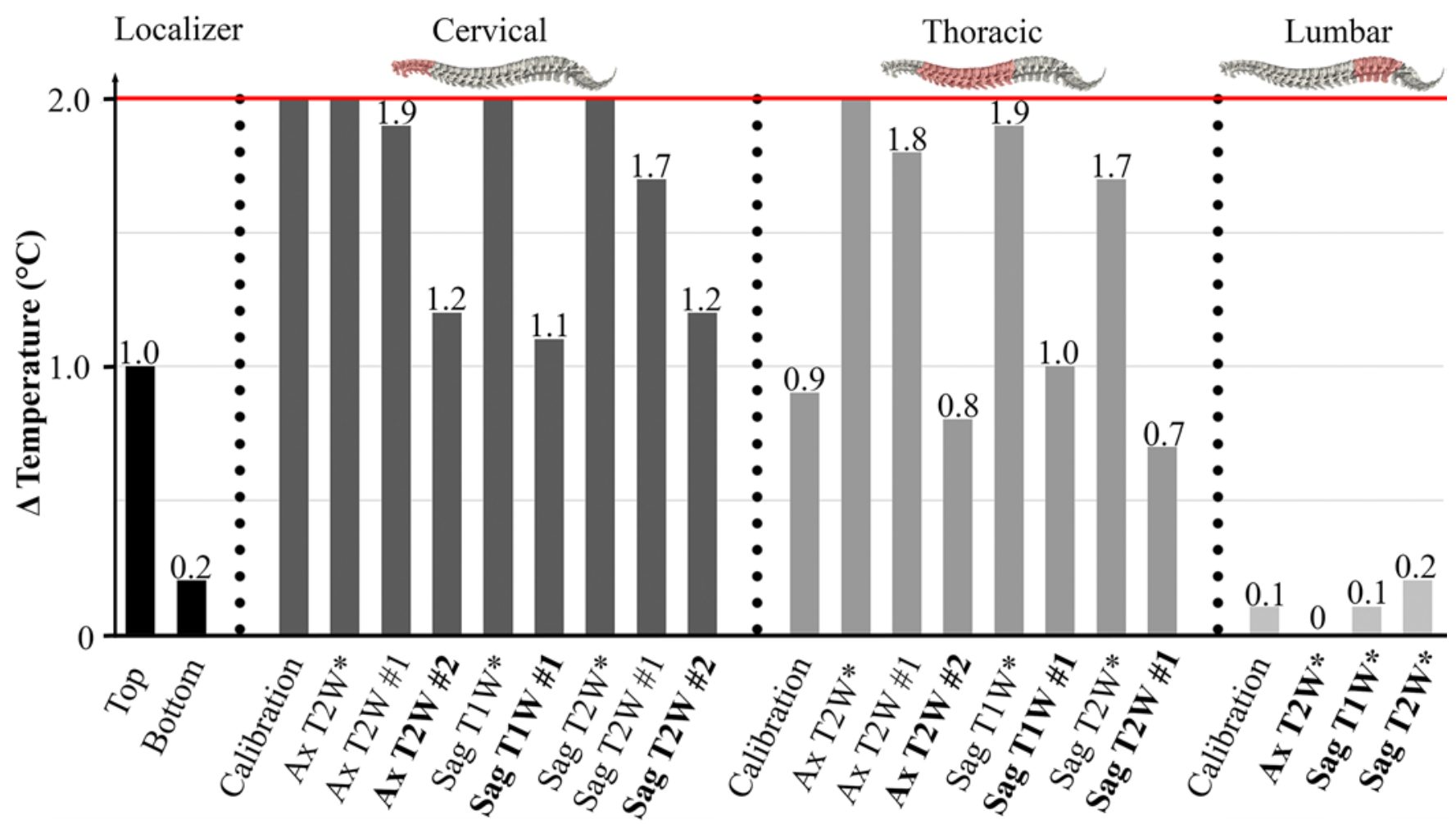

FIG. 3. Rises in temperature at the right electrode tips for localizer, C-, T-, and L-spine sequences are shown. When above (or approaching) a $2^{\circ} \mathrm{C}$ temperature rise, sequences were modified to reduce heating. The red line indicates a safe threshold of $2^{\circ} \mathrm{C}$. Sequences routinely used at our institution are identified by an asterisk $\left(^{*}\right)$ and bolded sequences represent those deemed to be safe for participants with DBS devices. Ax = axial; Sag = sagittal. Figure is available in color online only.

tions being for body MRI. ${ }^{10}$ Because the vast majority of MRI scans performed in patients with DBS currently constitute brain sequences (e.g., electrode localization) $)^{10,33}$ and because there is paucity of literature outlining safety and best practices for body MRI in DBS patients, ${ }^{6,17}$ work such as this is crucial to facilitate improved overall clinical care of DBS patients. Of note, as recently as 2009, nearly half of imaging centers were not performing brain MRI in DBS patients. ${ }^{33}$ It is not unreasonable to infer that these imaging centers would be even more uncomfortable performing body MRI. Expanding safety-related knowledge of MRI in patients with DBS devices, in particular body MRI, will minimize the chances that clinically necessary MRI studies are not performed based on what may be an extreme interpretation of the associated risks. ${ }^{7,22}$

Currently proposed MRI guidelines for DBS patients are highly restrictive, commonly imposing modifications on routine MRI protocols. These restrictions are in place to prevent additional MRI-related injuries. Modifications of routine MRI protocols typically require a specialized knowledge base (i.e., radiologists and physicists) and may result in increased acquisition time and compromised image quality. ${ }^{1,28}$ To decrease the SAR and B1+rms, and thus conform to guidelines, several options are available: reduced number of slices, echoes, and/or flip angles, as well as increased repetition time. ${ }^{1}$ Reducing the number of slices requires radiologist supervision to ensure the suspected problematic area is included in the field of view. This is

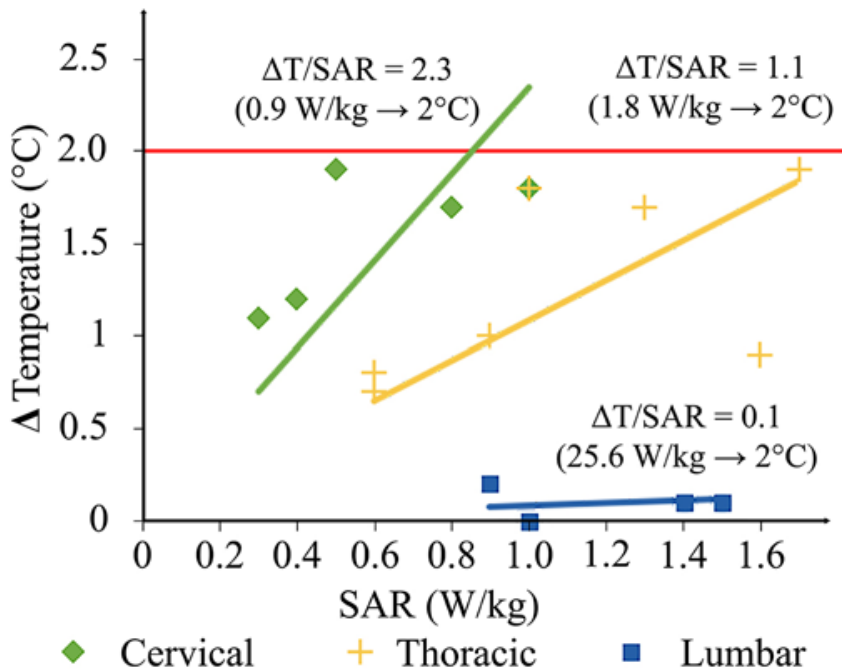

FIG. 4. The relationship between temperature increases and the SAR. Temperature rises and the associated SAR were plotted. The $\triangle T / S A R$ is represented with a linear relationship. The $\triangle T / S A R$ was greatest for the C-spine $\left(2.3^{\circ} \mathrm{C} /[\mathrm{W} / \mathrm{kg}]\right)$, followed by the T-spine $\left(1.1^{\circ} \mathrm{C} /[\mathrm{W} / \mathrm{kg}]\right)$. It was near 0 for the L-spine $\left(0.1^{\circ} \mathrm{C} /[\mathrm{W} / \mathrm{kg}]\right)$. Using this linear relationship, the SAR for a temperature increase of $2^{\circ} \mathrm{C}$ can be calculated: $0.9 \mathrm{~W} / \mathrm{kg}$ for the C-spine, $1.8 \mathrm{~W} / \mathrm{kg}$ for the T-spine, and $25.6 \mathrm{~W} / \mathrm{kg}$ for the L-spine $(25.6$ $\mathrm{W} / \mathrm{kg}$ ). The red line indicates a safe threshold of $2^{\circ} \mathrm{C}$. Note that the linear model assumed an intercept at 0 and excluded sequences for which a maximal temperature rise could not be defined because they were stopped once $2^{\circ} \mathrm{C}$ was reached. Figure is available in color online only. 
Boutet et al.

TABLE 5. The number of times sequences were acquired

\begin{tabular}{|c|c|c|c|c|c|c|c|c|c|}
\hline \multirow{2}{*}{$\begin{array}{c}\text { Case No. } \\
(n=9)\end{array}$} & \multicolumn{3}{|c|}{ Cervical Spine } & \multicolumn{3}{|c|}{ Thoracic Spine } & \multicolumn{3}{|c|}{ Lumbar Spine } \\
\hline & Axial T2W & Sagittal T2W & Sagittal T1W & Axial T2W & Sagittal T2W & Sagittal T1W & Axial T2W & Sagittal T2W & Sagittal T1W \\
\hline 1 & - & - & - & - & - & - & 2 & 2 & 2 \\
\hline 2 & 2 & 5 & 2 & - & - & - & - & 1 & - \\
\hline 3 & - & - & - & - & - & - & 2 & 4 & 3 \\
\hline 4 & - & - & - & - & - & - & 3 & 3 & 1 \\
\hline 5 & - & - & - & - & - & - & 2 & 4 & 2 \\
\hline 6 & - & 1 & - & - & 1 & - & 2 & 5 & - \\
\hline 7 & - & - & - & - & 1 & - & 1 & 4 & 1 \\
\hline 8 & - & - & - & - & - & - & 2 & 3 & - \\
\hline 9 & - & - & - & - & - & - & 2 & 4 & - \\
\hline Total & 2 & 6 & 2 & 0 & 2 & 0 & 16 & 30 & 9 \\
\hline
\end{tabular}

A total of 67 sequences were obtained.

particularly problematic for spine imaging to ensure appropriate levels are imaged. Autonomous technologistdriven MRI as is commonly employed in the clinical setting is consequently not possible. Alternatively, repetition time and flip angle may be modified to conform to vendor guidelines. However, they may affect image contrast, and the adjustment of these parameters is generally less practical and may be associated with technical difficulties. ${ }^{1,28}$ In summary, while DBS devices may be full-body eligible, performing body MRI in these patients according to vendor guidelines will usually prevent autonomous technologist-driven scanning.

The SARs of our spine protocols largely exceeded the recommended values. ${ }^{23}$ To decrease unacceptable tem-
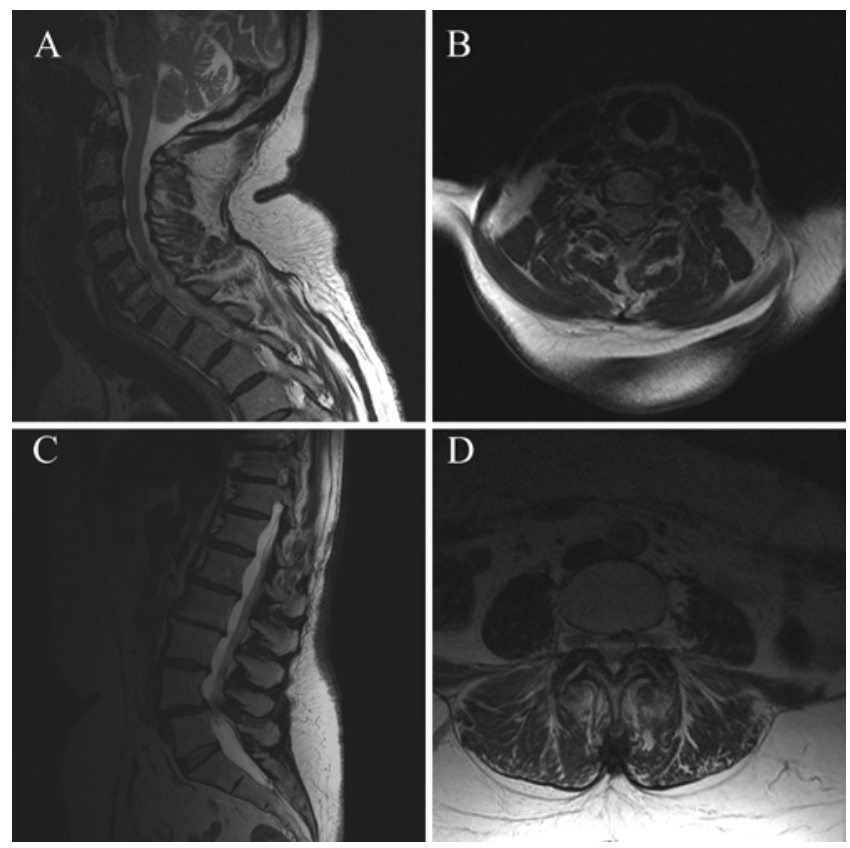

FIG. 5. Exemplar spine images. Sagittal ( $A$ and $C)$ and axial (B and D) T2W cervical ( $A$ and $B$ ) and lumbar ( $C$ and $D)$ MR images. perature increases, in addition to changing a 3D into a $2 \mathrm{D}$ sequence, we most commonly decreased the number of slices because insufficient anatomical coverage can be solved by repeated acquisition of spatially contiguous sequences. Changing the number of slices does not compromise image quality itself and may only add a few minutes of acquisition time to cover the required anatomical area. Importantly, although the number of slices had to be decreased compared to the routine spine protocols, this number was still higher than the number of slices that would have been included in protocols within the vendor guidelines. Having established spine MRI protocols with the highest slice numbers (i.e., body coverage) while maintaining safety will lead to greater technologist autonomy and will minimize the need for radiologist's supervision. Our safety data show, however, that the use of 3D sequences should generally not be entertained for DBS patients undergoing spine MRI.

Apart from the type of MRI hardware and parameters, and neuromodulation systems, DBS device configuration (i.e., geometry) is known to play a central role in device heating. 6,17 Positioning the DBS system off the isocen$\operatorname{ter}^{17}$ or with a rotational angle has each been shown to increase the overall measured temperatures in phantom studies. ${ }^{11,29,30}$ Similarly, investigators have commonly found larger temperature increases at the left compared to the right electrode tip; this may be owing to the different configuration of leads as the extension wire is most commonly tunneled under the right lateral side. Interestingly, we recorded larger temperature rises at the right electrode tips. Because heating at the right electrode tip was recorded during localizers and because the temperature probe location accuracy was ensured before and after the experiment, we think this finding is likely related to the interplay between the MRI and DBS devices. Our data highlighted the importance of the spatial relationship between the DBS device and the emitted RF pulses (and thus the coils): there was a clear difference in heating between the C- and L-spine, presumably related to the relationship between RF pulses and the device components included in the images. This finding is in line with the literature pertaining to other implants such as pacemakers. ${ }^{8}$ 
TABLE 6. Mean acquisition time per patient for $C$ - and L-spine sagittal T2W MRI

\begin{tabular}{cccc}
\hline & & \multicolumn{2}{c}{$\begin{array}{c}\text { Mean Acquisition Time Needed/ } \\
\text { Patient (mins)* }\end{array}$} \\
\cline { 3 - 4 } Pulse Sequence & $\begin{array}{c}\text { No. of } \\
\text { Patients }\end{array}$ & $\begin{array}{c}\text { Phantom-Based } \\
\text { Safety Protocol }\end{array}$ & $\begin{array}{c}\text { Routine Clinical } \\
\text { Protocol }\end{array}$ \\
\hline C-spine sagittal T2W & 1 & 8.5 & 3.2 \\
\hline L-spine sagittal T2W & 8 & $10.8 \pm 2.7$ & 4.6 \\
\hline
\end{tabular}

For the phantom-based safety protocol, acquisition times for C- and L-spine sagittal T2W MRI were 1.7 and 3 minutes, respectively. The mean acquisition time per patient was determined by summing the acquisition time of each repeat image acquisition. For example, case 1 (see Table 5 ) required two Lspine sagittal T2W images to cover the full anatomy $(2 \times 3=6$ minutes). Eight patients underwent full coverage of L-spine on sagittal T2W images (cases 1 and 3-9 [Table 5]), and 1 patient underwent full coverage of $\mathrm{C}$-spine sagittal T2W (case 2 [Table 5]). The patient in case 2 (Table 5) also underwent one L-spine sagittal T2W imaging sequence to acquire screening images (rather than full anatomical coverage) and was not included in the L-spine sagittal T2W group. Clinical protocols on patients without DBS devices require one sequence acquisition to cover the required spine anatomy.

* Values are presented as the mean acquisition time or as the mean acquisition time \pm SD.

Alternatively, different body landmarks used for the spine segments changes the proximity to coil rung elements and capacitors, which may induce high local SARs. ${ }^{34}$ Previous studies have also found a linear relationship between the SAR and temperature rise; however, the $\Delta \mathrm{T} / \mathrm{SAR}$ was inconsistent across different MRI hardware combinations. ${ }^{3,4}$ Similarly, although we showed linear $\Delta \mathrm{T} / \mathrm{SARs}$, they were different across the spine segments being scanned. As suggested in previous studies, $3,4,6,18$ these general indicators of heating are unreliable for predicting heating for specific MRI hardware and DBS device models/configurations.

\section{Study Limitations}

Our study has a number of limitations. Although a large number of spine sequences $(n=67)$ were acquired overall, 9 different patients were scanned. Also, acute intracranial findings immediately adjacent to the DBS leads may have been masked by the metallic artifact. As stated previously in similar reports, phantom studies have a number of limitations. ${ }^{6}$ Most importantly, our safety data are not generalizable to other MRI hardware or institutions. However, with careful planning and the appropriate expertise, other institutions may replicate our experiment without substantial costs. Similarly, findings in this study are not generalizable to other magnetic field strengths (e.g., $3 \mathrm{~T}$ ) and other implants (e.g., spinal cord stimulators). Furthermore, since our MRI software did not compute B1+rms, our phantom safety data were based on SARs only. As B1+rms was recently incorporated into the vendor guidelines, it would have been preferable to also compute this measure of heating. Finally, given the adoption of one manufacturer only (i.e., Medtronic), our results are difficult to generalize to the other DBS companies.

Guidelines have been developed to assess the MRI-related safety of medical implants., ${ }^{216}$ These guidelines may allow increased safety data generalizability across MRI systems and institutions. As patients with DBS systems are increasing in number, clinicians are often faced with clinical queries in this patient population that are best assessed using MRI. While waiting for updated vendor guidelines is the optimal option for patient safety, it also has negative impact on patient care as it limits the use of an important diagnostic imaging modality. As with most aspects of medicine, clinicians should always weigh the advantages and disadvantages of a diagnostic test. If MRI is likely to provide significant benefits for the patient with DBS, then assumption of some degree of risk may be acceptable. ${ }^{22}$

\section{Conclusions}

We safely acquired spine MR images in patients with fully internalized DBS systems using a protocol outside the prescribed vendor safety guidelines. This MRI protocol was predicated on local phantom safety data, which maximized the number of acquired slices compared to guideline-consistent MRI protocols, thus minimizing the need for radiologists to continually monitor acquired images. Our phantom safety data also clearly demonstrated a safety gradient among the different spine segments. As a large proportion of patients with DBS are likely to be denied clinically indicated MRI, this study is intended to share our experience performing spine MRI that is required for the management of spine disorders in a large proportion of these patients. We hope this study will prompt other groups to confirm and build on our findings, such that MRI use in DBS patients might be expanded.

\section{Acknowledgments}

We would like to acknowledge Eric Fiveland for his technical expertise and Hadiqa Malik for her help with the research ethics proposal.

\section{References}

1. Allison J, Yanasak N: What MRI sequences produce the highest specific absorption rate (SAR), and is there something we should be doing to reduce the SAR during standard examinations? AJR Am J Roentgenol 205:W140, 2015

2. ASTM International: ASTM F2052-15: Standard Test Method for Measurement of Magnetically Induced Displacement Force on Medical Devices in the Magnetic Resonance Environment. West Conshohocken, PA: ASTM International, 2015 (https://www.astm.org/Standards/F2052. htm) [Accessed January 3, 2020]

3. Baker KB, Tkach JA, Nyenhuis JA, Phillips M, Shellock FG, Gonzalez-Martinez J, et al: Evaluation of specific absorption rate as a dosimeter of MRI-related implant heating. J Magn Reson Imaging 20:315-320, 2004

4. Baker KB, Tkach JA, Phillips MD, Rezai AR: Variability in RF-induced heating of a deep brain stimulation implant across MR systems. J Magn Reson Imaging 24:1236-1242, 2006

5. Boston Scientific: ImageReady MRI Guidelines for Boston Scientific Deep Brain Stimulation Systems. Marlborough, MA: Boston Scientific, 2017

6. Boutet A, Hancu I, Saha U, Crawley A, Xu DS, Ranjan M, et al: 3-Tesla MRI of deep brain stimulation patients: safety assessment of coils and pulse sequences. J Neurosurg 132:586-594, 2020 
7. Boutet A, Rashid T, Hancu I, Elias GJB, Gramer RM, Germann J, et al: Functional MRI safety and artifacts during deep brain stimulation: experience in 102 patients. Radiology 293:174-183, 2019

8. Calcagnini G, Triventi M, Censi F, Mattei E, Bartolini P, Kainz $\mathrm{W}$, et al: In vitro investigation of pacemaker lead heating induced by magnetic resonance imaging: role of implant geometry. J Magn Reson Imaging 28:879-886, 2008

9. Deli G, Balas I, Nagy F, Balazs E, Janszky J, Komoly S, et al: Comparison of the efficacy of unipolar and bipolar electrode configuration during subthalamic deep brain stimulation.

Parkinsonism Relat Disord 17:50-54, 2011

10. Falowski S, Safriel Y, Ryan MP, Hargens L: The rate of magnetic resonance imaging in patients with deep brain stimulation. Stereotact Funct Neurosurg 94:147-153, 2016

11. Georgi JC, Stippich C, Tronnier VM, Heiland S: Active deep brain stimulation during MRI: a feasibility study. Magn Reson Med 51:380-388, 2004

12. Gupte AA, Shrivastava D, Spaniol MA, Abosch A: MRIrelated heating near deep brain stimulation electrodes: more data are needed. Stereotact Funct Neurosurg 89:131-140, 2011

13. Hancu I, Boutet A, Fiveland E, Ranjan M, Prusik J, Dimarzio $\mathrm{M}$, et al: On the (non-)equivalency of monopolar and bipolar settings for deep brain stimulation fMRI studies of Parkinson's disease patients. J Magn Reson Imaging 49:1736-1749, 2019

14. Hariz M: My 25 stimulating years with DBS in Parkinson's disease. J Parkinsons Dis 7 (s1):S33-S41, 2017

15. Henderson JM, Tkach J, Phillips M, Baker K, Shellock FG, Rezai AR: Permanent neurological deficit related to magnetic resonance imaging in a patient with implanted deep brain stimulation electrodes for Parkinson's disease: case report. Neurosurgery 57:E1063, 2005

16. International Organization for Standardization: ISO/TS 10974:2018(E): Assessment of the Safety of Magnetic Resonance Imaging for Patients With an Active Implantable Medical Device. Geneva: International Organization for Standardization, 2018

17. Kahan J, Papadaki A, White M, Mancini L, Yousry T, Zrinzo $\mathrm{L}$, et al: The safety of using body-transmit MRI in patients with implanted deep brain stimulation devices. PLoS One 10:e0129077, 2015

18. Kainz W: MR heating tests of MR critical implants. J Magn Reson Imaging 26:450-451, 2007

19. Kainz W, Neubauer G, Uberbacher R, Alesch F, Chan DD: Temperature measurement on neurological pulse generators during MR scans. Biomed Eng Online 1:2, 2002

20. Larson PS, Richardson RM, Starr PA, Martin AJ: Magnetic resonance imaging of implanted deep brain stimulators: experience in a large series. Stereotact Funct Neurosurg 86:92-100, 2008

21. Lozano AM, Lipsman N, Bergman H, Brown P, Chabardes S, Chang JW, et al: Deep brain stimulation: current challenges and future directions. Nat Rev Neurol 15:148-160, 2019

22. Martin AJ: MRI in patients with deep brain stimulation electrodes: balancing risks and benefits. Radiology 293:184185, 2019

23. Medtronic: MRI Guidelines for Medtronic Deep Brain Stimulation Systems. Minneapolis: Medtronic, 2015 (http:// www.mriquestions.com/uploads/3/4/5/7/34572113/dbs medtronics_contrib_228155.pdf) [Accessed January 3, 2020]

24. Ozdoba C, Gralla J, Rieke A, Binggeli R, Schroth G: Myelography in the age of MRI: why we do it, and how we do it. Radiol Res Pract 2011:329017, 2011

25. Pötter M, Herzog J, Siebner HR, Kopper F, Steigerwald F, Deuschl G, et al: Subthalamic nucleus stimulation modulates audiospinal reactions in Parkinson disease. Neurology 70:1445-1451, 2008
26. Sammartino F, Krishna V, Sankar T, Fisico J, Kalia SK, Hodaie M, et al: 3-Tesla MRI in patients with fully implanted deep brain stimulation devices: a preliminary study in 10 patients. J Neurosurg 127:892-898, 2017

27. Sandow BA, Donnal JF: Myelography complications and current practice patterns. AJR Am J Roentgenol 185:768771,2005

28. Schmitz BL, Aschoff AJ, Hoffmann MH, Grön G: Advantages and pitfalls in 3T MR brain imaging: a pictorial review. AJNR Am J Neuroradiol 26:2229-2237, 2005

29. Shrivastava D, Abosch A, Hanson T, Tian J, Gupte A, Iaizzo PA, et al: Effect of the extracranial deep brain stimulation lead on radiofrequency heating at 9.4 Tesla (400.2 MHz). J Magn Reson Imaging 32:600-607, 2010

30. Shrivastava D, Abosch A, Hughes J, Goerke U, DelaBarre L, Visaria R, et al: Heating induced near deep brain stimulation lead electrodes during magnetic resonance imaging with a 3 T transceive volume head coil. Phys Med Biol 57:5651-5665, 2012

31. Spiegel J, Fuss G, Backens M, Reith W, Magnus T, Becker $\mathrm{G}$, et al: Transient dystonia following magnetic resonance imaging in a patient with deep brain stimulation electrodes for the treatment of Parkinson disease. Case report. J Neurosurg 99:772-774, 2003

32. St. Jude Medical: MRI Procedure Information for St. Jude Medical MR Conditional Deep Brain Stimulation Systems. Little Canada, MN: St. Jude Medical, 2018 (https:// manuals.sjm.com/Search-Form?re=North-America\&cc=U $\mathrm{S} \& \ln =$ all $\&$ ry $=\mathrm{SJM}+\mathrm{rin} \mathrm{+}+\& \mathrm{ipp}=10 \& \mathrm{Page}=1)[$ Accessed January 3, 2020]

33. Tagliati M, Jankovic J, Pagan F, Susatia F, Isaias IU, Okun MS: Safety of MRI in patients with implanted deep brain stimulation devices. Neuroimage 47 (Suppl 2):T53-T57, 2009

34. Yeo DT, Wang Z, Loew W, Vogel MW, Hancu I: Local specific absorption rate in high-pass birdcage and transverse electromagnetic body coils for multiple human body models in clinical landmark positions at 3T. J Magn Reson Imaging 33:1209-1217, 2011

35. Zrinzo L, Yoshida F, Hariz MI, Thornton J, Foltynie T, Yousry TA, et al: Clinical safety of brain magnetic resonance imaging with implanted deep brain stimulation hardware: large case series and review of the literature. World Neurosurg 76:164-172, 69-73, 2011

\section{Disclosures}

This work was supported by the German Research Foundation (Deutsche Forschungsgemeinschaft, DFG NE 2276/1-1) with funding to C.N., the RR Tasker Chair in Functional Neurosurgery at University Health Network, and a Tier 1 Canada Research Chair in Neuroscience. A.B. was responsible for the data analysis. The corresponding author confirms that he had full access to all the data in the study and had final responsibility for the decision to submit for publication.

I.H. is a National Institutes of Health (NIH) employee. The opinions expressed in this article are the authors' own and do not reflect the views of the NIH, the Department of Health and Human Services, or the United States government. B.L. is now a Medtronic employee; he was not at the time of the completion of this work. Medtronic had no role in data acquisition, analysis, or interpretation. A.F. reports grants, personal fees, and nonfinancial support from Abbvie and Medtronic; grants and personal fees from Boston Scientific; personal fees from Sunovion, Chiesi farmaceutici, and UCB; and grants and personal fees from Ipsen, outside the submitted work. S.K.K. reports an honorarium and consulting fees from Medtronic. A.M.L. serves as a consultant for Medtronic, Abbott, Boston Scientific, St. Jude, and Functional Neuromodulation. 


\section{Author Contributions}

Conception and design: Lozano, Boutet, Valencia, Fasano, Munhoz. Acquisition of data: Boutet, Elias, Gramer, Naheed, Bennett, Li, Gwun, Chow, Maciel, Foltz. Analysis and interpretation of data: Lozano, Boutet, Elias, Gramer, Neudorfer, Gwun, Mikulis, Hancu, Kucharczyk. Drafting the article: Boutet. Critically revising the article: all authors. Reviewed submitted version of manuscript: all authors. Statistical analysis: Boutet, Germann. Administrative/technical/material support: Lozano, Kucharczyk. Study supervision: Lozano.

\section{Correspondence}

Andres M. Lozano: Toronto Western Hospital, Toronto, ON, Canada.lozano@uhnresearch.ca. 